\title{
UAV AIRCRAFT MODEL FOR CONTROL SYSTEM FAILURES ANALYSIS
}

\author{
Marcin Żugaj, Przemyseaw Bibik, Mariusz Jacewicz \\ Warsaw University of Technology, Institute of Aeronautics and Applied Mechanics, Warszawa, Poland \\ e-mail: zugaj@meil.pw.edu.pl;pbibik@meil.pw.edu.pl; mjacewicz@meil.pw.edu.pl
}

\begin{abstract}
In the paper, influence of control surface failures on UAV aircraft dynamics is investigated. A method for control loads determination for a nonlinear UAV aircraft model is presented. The model has been developed to analyse the influence of various control surface failures on aircraft controllability and to form the background for developing reconfiguration methods of flight control systems. The analysis of the control system failure impact on the aircraft dynamics and the ability of the control system to reconfiguration are presented.
\end{abstract}

Keywords: UAV, flight dynamics, control, reconfiguration

\section{Introduction}

In the last few decades, the number of unmanned aircraft operations has increased. At the beginning UAVs were mainly used for the purposes of military forces. Today, plenty of different types of Unmanned Aircraft Vehicles (UAVs) perform missions which are too dull, dirty, or dangerous for manned aircraft also in the civil airspace. The main problems which slow down the spreading of the UAVs in the civil sector is the integration of Unmanned Aerial Systems (UAS) with the Air Traffic Management systems and safety of the UAS. For the manned aviation, there are plenty of different regulations which are forcing the manufacturers and operators to enhance safety and reliability of the aircraft. At the time, there are no such regulations for unmanned systems. Different sources show how hazardous the current unmanned systems are. Some reports show that the rate of UAV accidents is about 32 per 100000 flight hours, which is 3200 times more than the number for commercial liner aircraft (Defense Science Board Study, 2004). These numbers show that there is much work to do within the safety area of the unmanned systems. Various institutions around the world are now focusing on the safety aspects of the UAS usage (Loh et al., 2006, 2009; Uhlig et al., 2006; Lin et al., 2014). The researchers try to convince manufacturers that the safety must be taken into account from the very beginning of the development process and that it does not have to increase the cost of the system very much. Especially hazardous are designs that are based on the COTS elements and subsystems. It is well known that the total safety of a complex system depends on the safety of each element. However, there are ways to assure that the failure of the single element or subsystem will not lead to an accident.

The UAV aircraft safety depends on several unpredictable factors such as, for instance, hostile actions both inside and outside the aircraft. When failures occur, the most important actions must be aimed at maintaining the aircraft controllability. The UAV aircrafts perform their flight mainly using autopilots. The automatic flight control systems are designed for normal operation and may not be able to react sufficiently efficient when an unpredicted malfunction appears. A method for assuring safety in the case of unpredictable failure is reconfiguration of the flight control system (Kozak et al., 2014), which would make the control system fault tolerant and ensure aircraft controllability in the event of fault. 
The process of flight control system reconfiguration is aimed to take advantage of the working part of the control system in the case of partial system failure. The reconfiguration can be performed in three levels. Level 1 performs two functions: sets control surfaces to compensate the failure effects and corrects the strategy of control surfaces handling (Burcham, 1997). In level 2, an attempt is made to rearrange the autopilot control laws to adopt to a new situation, (Bodson, 2003; Hass and Wells, 2003). In level 3, on the basis of prediction about the future situation, refinement of the flight trajectory is performed (Masui et al., 2004; Suzuki et al., 2004).

Typically, control surfaces such as elevators, ailerons and flaps work in pairs and are located in opposite sides of the longitudinal plane of symmetry of an aircraft (Fig. 1). This configuration is easy to handle but its redundancy is highly limited. The redundancy can be increased by splitting all paired control surfaces and control them individually. This feature of the control system property is named structural redundancy (Burcham, 1997; Żugaj and Narkiewicz, 2009) and results in increasing the flexibility of aircraft handling. The decoupled control system is more reliable for the reconfiguration process and can be supported by a broad scope of reconfiguration schemes.

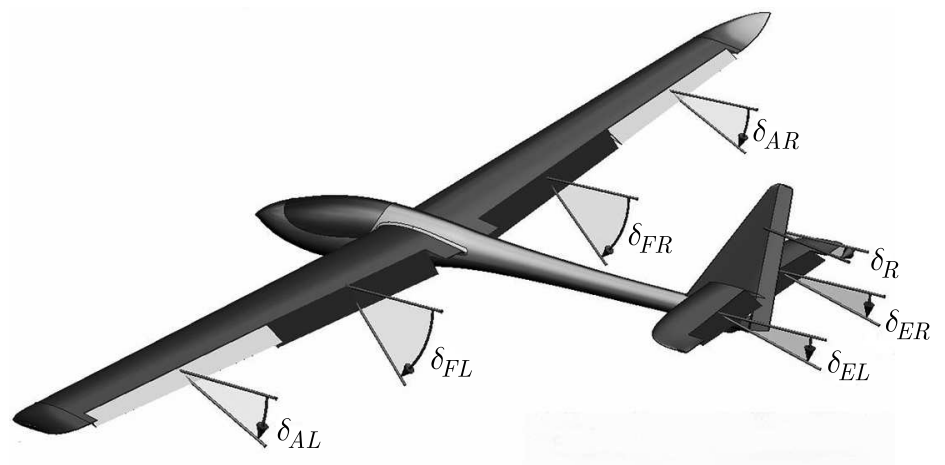

Fig. 1. Control surfaces layout

The dynamic model of UAV is needed for analysis and synthesis of the control system reconfiguration method at each level. That is why the model must assure simulation of each control surface failure and handling strategy (Żugaj and Narkiewicz, 2007, 2010). It involves complex modeling of control loads where all control surfaces are considered individually, not in pairs as usually.

The aim of the present work is to develop a UAV dynamic model for analysis of reconfiguration of the flight control system. A six degrees of freedom nonlinear model with decoupled control surfaces has been derived using the classical approach. The contributions of each control surface to aerodynamic loads are estimated based on distributions of lift and drag forces along lifting surfaces span due to the control surface deflection obtained from CFD software. The model is used for investigation of UAV aircraft performances under control surfaces failure. The analysis of decoupled control system efficiency for reconfiguration has been performed as well.

\section{UAV nonlinear model}

The UAV airplane considered in this study is shown in Fig. 1. The airplane is modeled as a rigid body with six degrees of freedom. The control forces and moments are produced by two ailerons and two flaps placed on the wing trailing edge, two elevators placed on the horizontal stabilizer trailing edge, and the rudder placed on the trailing edge of the fin. The aircraft is propelled by one electric motor with a constant pitch tracking propeller placed in front of the fuselage.

The elevators, ailerons and flaps work in pairs and the operation of each pair is modeled as a single control input. The modeling of the control loads of individual control surfaces is 
required due to the UAV model application in analysis and validation of the control system reconfiguration. Each control surface is treated as an individual control input. So, the number of control inputs increase from four in the classical to seven in the reconfigurable configuration.

The aircraft equations of motion are derived in the body co-ordinate system $O_{b} x_{b} y_{b} z_{b}$ (Fig. 2) fixed to the airplane fuselage. The centre $O_{b}$ of the system is placed at the UAV gravity centre. The $O_{b} x_{b}$ axis lies in the plane of aircraft symmetry and is directed forward. The $O_{b} y_{b}$ axis is perpendicular to the aircraft plane of symmetry and points right, the $O_{b} z_{b}$ axis points "down".

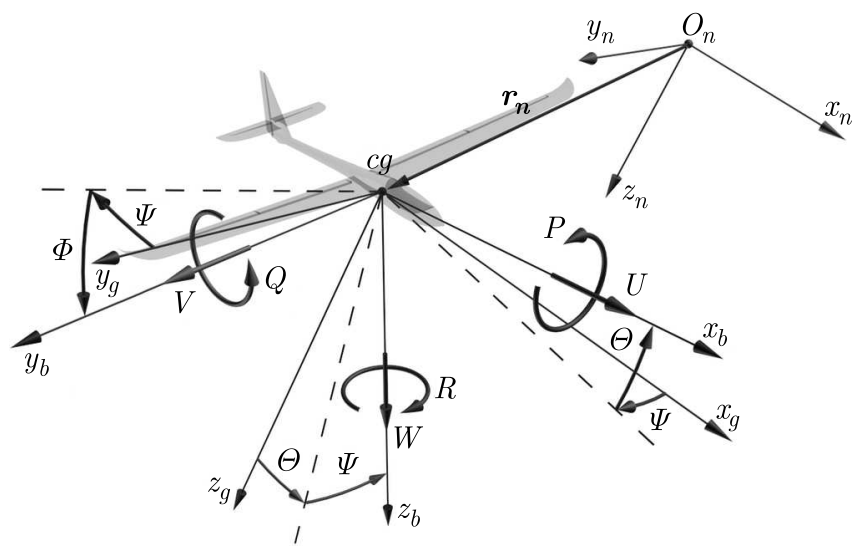

Fig. 2. Coordinate systems

The aircraft translations and attitude angles are calculated in the inertial co-ordinate system $O_{n} x_{n} y_{n} z_{n}$; the centre of this system $O_{n}$ is placed at an arbitrary point on the earth surface. The $O_{n} z_{n}$ axis is along the vector of gravity acceleration, points down. The $O_{n} x_{n} z_{n}$ plane is horizontal, tangent to the earth surface, the $O_{n} x_{n}$ axis points to the North and $O_{n} y_{n}$ axis to the East.

The vector $\mathbf{y}=\left[\begin{array}{llllll}x_{n} & y_{n} & z_{n} & \Phi & \Theta & \Psi\end{array}\right]^{\mathrm{T}}$ defines position and attitude of the aircraft (Fig. 2). It is composed of the vector of the aircraft position $\mathbf{r}_{n}=\left[\begin{array}{lll}x_{n} & y_{n} & z_{n}\end{array}\right]^{\mathrm{T}}$ in the ground system of co-ordinates $O_{n} x_{n} y_{n} z_{n}$ and the roll $\Phi$, pitch $\Theta$ and yaw $\Psi$ angles describing the aircraft attitude. The airplane state vector $\mathbf{x}=\left[\begin{array}{ll}\mathbf{v} & \boldsymbol{\omega}\end{array}\right]^{\mathrm{T}}$ is composed of linear velocity components $\mathbf{v}=\left[\begin{array}{lll}U & V & W\end{array}\right]^{\mathrm{T}}$ and the angular rate $\boldsymbol{\omega}=\left[\begin{array}{lll}P & Q & R\end{array}\right]^{\mathrm{T}}, P, Q, R$ - angular velocities.

The vectors of the aircraft state, position and attitude are related by a kinematic equation

$$
\dot{\mathbf{y}}=\mathbf{T x}
$$

The matrix $\mathbf{T}$ is composed of two matrices: $\mathbf{T}_{V}$ relating to velocities and $\mathbf{T}_{\Omega}$ - to rates

$$
\mathbf{T}=\left[\begin{array}{cc}
\mathbf{T}_{V} & \mathbf{0} \\
\mathbf{0} & \mathbf{T}_{\Omega}
\end{array}\right]
$$

where

$$
\begin{aligned}
\mathbf{T}_{V} & =\left[\begin{array}{ccc}
\cos \Theta \cos \Psi & \sin \Theta \sin \Phi \cos \Psi-\cos \Phi \sin \Psi & \cos \Phi \sin \Theta \cos \Psi+\sin \Phi \sin \Psi \\
\cos \Theta \sin \Psi & \sin \Theta \sin \Phi \sin \Psi+\cos \Phi \cos \Psi & \cos \Phi \sin \Theta \sin \Psi-\sin \Phi \cos \Psi \\
-\sin \Theta & \sin \Phi \cos \Theta & \cos \Phi \cos \Theta
\end{array}\right] \\
\mathbf{T}_{\Omega} & =\left[\begin{array}{ccc}
1 & \sin \Phi \tan \Theta & \cos \Phi \tan \Theta \\
0 & \cos \Phi & -\sin \Phi \\
0 & \sin \Phi \sec \Theta & \cos \Phi \sec \Theta
\end{array}\right]
\end{aligned}
$$

The airplane equations of motion have been obtained by summing up forces and moments from inertia, gravity $\mathbf{f}_{G}$, aerodynamic $\mathbf{f}_{A}$, and propulsion $\mathbf{f}_{T}$ loads (Nizioł, 2005)

$$
\mathbf{A} \dot{\mathbf{x}}+\mathbf{B}(\mathbf{x}) \mathbf{x}=\mathbf{f}_{A}(\mathbf{x}, \mathbf{y}, \boldsymbol{\delta})+\mathbf{f}_{G}(\mathbf{y})+\mathbf{f}_{T}\left(\mathbf{x}, \mathbf{y}, \delta_{T}\right)
$$


where $\mathbf{A}$ is the aircraft inertia matrix, $\mathbf{B}$ - gyroscopic matrix, $\boldsymbol{\delta}$ - vector of control surface deflections and $\delta_{T}$ is the position of the throttle lever

$$
\boldsymbol{\delta}=\left[\begin{array}{lllllll}
\delta_{A R} & \delta_{A L} & \delta_{E R} & \delta_{E L} & \delta_{R} & \delta_{F R} & \delta_{F L}
\end{array}\right]^{\mathrm{T}}
$$

The first letter of the subscripts $A, E, F, R$ denotes the type of the control surface, i.e. aileron, elevator, flap and rudder, respectively. The second letter denotes the position of the control surface: $L$ - left, $R$ - right.

The matrix $\mathbf{A}$ describes inertia properties of the aircraft, and the matrix $\mathbf{B}(\mathbf{x})=\boldsymbol{\Omega}(\mathbf{x}) \mathbf{A}$ results from inertia loads not depending on accelerations (Nizioł, 2005)

$$
\mathbf{A}=\left[\begin{array}{cccccc}
m & 0 & 0 & 0 & 0 & 0 \\
0 & m & 0 & 0 & 0 & 0 \\
0 & 0 & m & 0 & 0 & 0 \\
0 & 0 & 0 & I_{x} & 0 & -I_{x z} \\
0 & 0 & 0 & 0 & I_{y} & 0 \\
0 & 0 & 0 & -I_{x z} & 0 & I_{z}
\end{array}\right] \quad \boldsymbol{\Omega}(\mathbf{x})=\left[\begin{array}{cccccc}
0 & -R & Q & 0 & 0 & 0 \\
R & 0 & -P & 0 & 0 & 0 \\
-Q & P & 0 & 0 & 0 & 0 \\
0 & -W & V & 0 & -R & Q \\
W & 0 & -U & R & 0 & -P \\
-V & U & 0 & -Q & P & 0
\end{array}\right]
$$

where $m$ is the aircraft mass, $I_{x}, I_{y}, I_{z}$ are moments of inertia, and $I_{x z}$ is the product of inertia.

The aerodynamic loads $\mathbf{f}_{A}$ may be expressed as the sum of two parts

$$
\mathbf{f}_{A}(\mathbf{x}, \mathbf{y}, \boldsymbol{\delta})=\mathbf{f}_{A S}(\mathbf{x}, \mathbf{y})+\mathbf{f}_{\delta}(\mathbf{x}, \mathbf{y}, \boldsymbol{\delta})
$$

The part $\mathbf{f}_{\delta}(\mathbf{x}, \mathbf{y}, \boldsymbol{\delta})$ depends on deflections of control surfaces and the part $\mathbf{f}_{A S}(\mathbf{x}, \mathbf{y})$ does not.

Substituting Eq. (2.7) into (2.4), rearranging and multiplying by $\mathbf{A}^{-1}$, the nonlinear aircraft model can be written as

$$
\dot{\mathbf{x}}=\mathbf{f}_{1}(\mathbf{x}, \mathbf{y})+\mathbf{f}_{2}(\mathbf{x}, \mathbf{y}, \boldsymbol{\delta})+\mathbf{f}_{3}\left(\mathbf{x}, \mathbf{y}, \delta_{T}\right)
$$

In Eq. (2.8), the first component does not depend on aircraft control, the second one describes airplane loads increment due to deflection of the control surface, and the third one describes airplane loads due to thrust control.

The point of interest in this study is the increment of aerodynamic loads produced by deflection of asymmetric (individual) control surfaces. The control surfaces are modeled as trailing edge plain flaps (Young, 1953). The aerodynamic loads depend on the lifting surface outline and section (airfoil) geometry (Fig. 3). The flap deflection changes the lift and drag forces around the part of the lifting surface where the flap is located. The lift and drag forces as well as the pitching moment can be expressed in form (Cook, 2007)

$$
L=\bar{q} S C_{L} \quad D=\bar{q} S C_{D} \quad M=\bar{q} S c C_{m}
$$

where $\bar{q}$ is the free stream dynamic pressure, $S$ is the lifting surface plan form area, $c$ is the airfoil chord $C_{L}, C_{D}$ and $C_{m}$ are lifting surface lift, drag and pitching moment coefficients, respectively. The airfoil lift increment due to plain flap deflection results from effective change of the airfoil camber and airfoil angle of attack $\alpha^{\prime}$ (Young, 1953). The lift coefficient increment depends on the chord $c_{f}$, span $b_{f}$ and deflection angle $\delta$ of the flap (Fig. 3).

The airfoil drag increment results from profile drag increment which can be analyzed in the same way as the lift coefficient increment. The profile drag does not have a significant influence on the lifting surface drag regarding to the induced drag. The lifting surface induced drag increment due to flap deflection can be estimated by (Young, 1953)

$$
\Delta C_{D i}=K \frac{\Delta C_{L \delta}^{2}}{\pi A}
$$




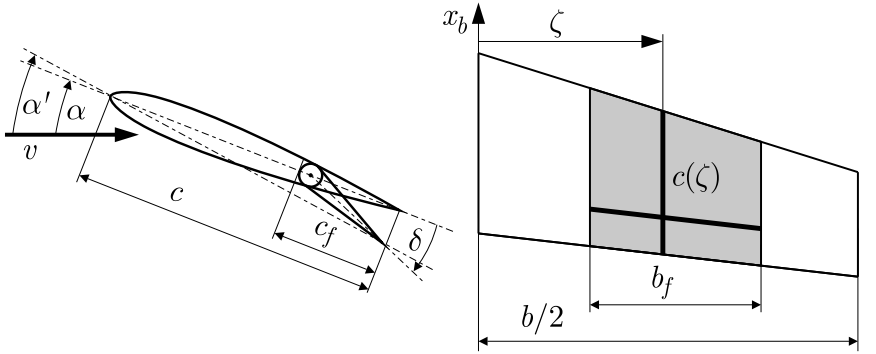

Fig. 3. Section and lifting surface parameters

where $\Delta C_{L \delta}$ is the lifting surface lift coefficient increment due to flap deflection, $A$ is the lifting surface aspect ratio and $K$ is an empirical constant.

The analytical methods for aerodynamic coefficients estimation allow obtaining only quantities of lift, drag and moment increments. These increment distributions along the lifting surface are also needed to calculate the influence of the flap deflection on the aircraft aerodynamics roll and yaw moments.

The numerical analysis of the lift increment distribution due to flap deflection have been done using a free software. The calculations have been performed for isolated lifting surfaces at cruise flight conditions of the UAV aircraft. An example of the wing lift coefficient increments due to aileron and trailing edge flap deflection and the horizontal tail lift coefficient increment due to elevator deflection in the body coordinate system are presented in Figs. 4-6.

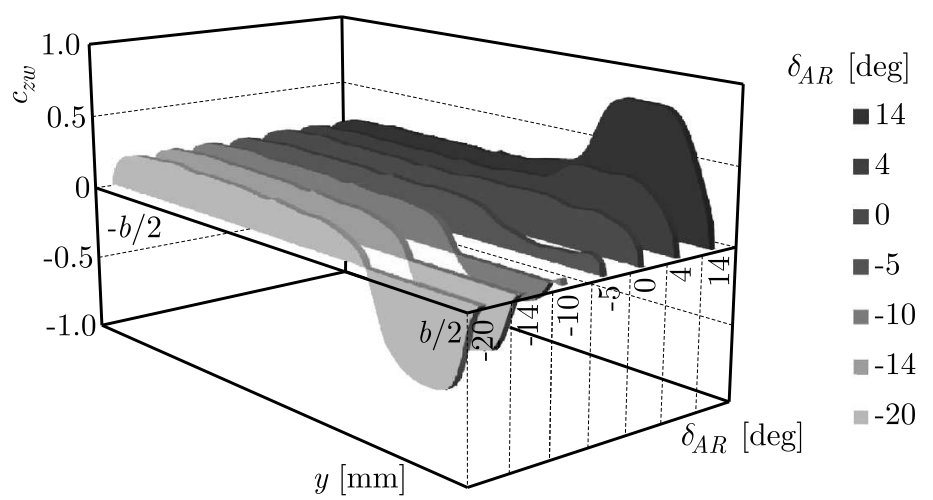

Fig. 4. Wing lift coefficient distribution due to aileron deflection

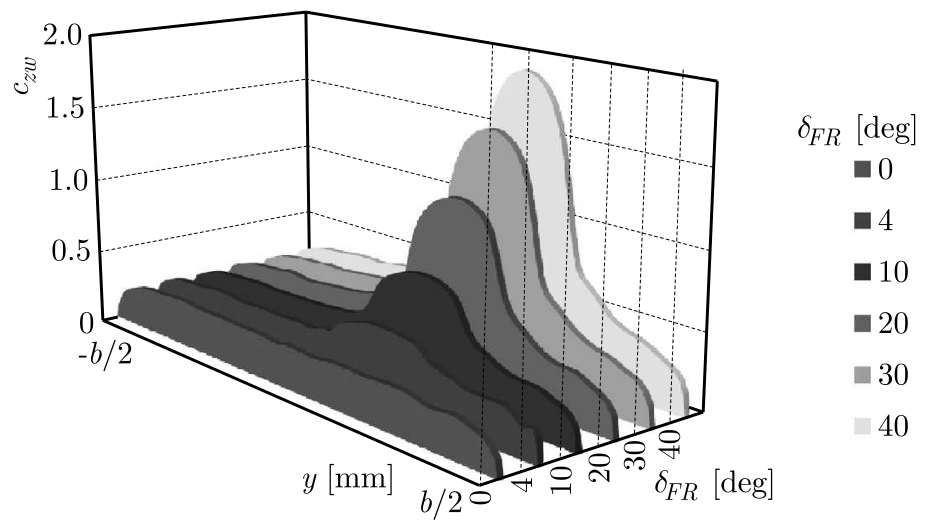

Fig. 5. Wing lift coefficient distribution due to flap deflection 


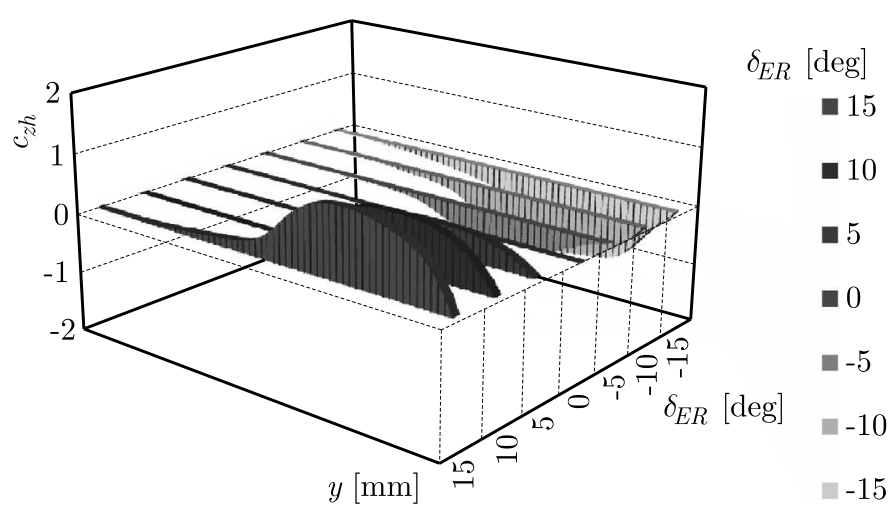

Fig. 6. Horizontal tail lift coefficient distribution due to elevator deflection

The control derivatives for each control surface have been obtained based on the lift distribution. The increment of the lift $\Delta c_{L i j}(\zeta)$, drag $\Delta c_{D i j}(\zeta)$ and pitching moment $\Delta c_{m i j}(\zeta)$ coefficients distribution of the $i$-th control surface and $j$-th deflection angle along the appropriate lifting surface span have been calculated as differences of the distribution for the clear and $j$-th flap configuration at first. Next, the control derivatives have been obtained using analytical methods presented in Cook (2007) and Nizioł (2005) as an integral in general form

$$
\Delta f_{2 i j}=\bar{q} \int_{0}^{b / 2} g\left(c(\zeta), \Delta c_{L i j}(\zeta), \Delta c_{D i j}(\zeta), \Delta c_{m i j}(\zeta), \zeta\right) d \zeta
$$

The $i$-th increment of the control loads $\Delta \mathbf{f}_{2 i}\left(\mathbf{x}, \mathbf{y}, \delta_{i}\right)$ as functions of the flight condition parameters (angle of attack, airspeed, etc.) and the $i$-th control surface deflection angle have been formed by combining the control derivatives for all deflection angles of the $i$-th control surface. The modeled aerodynamic loads can be written in the form

$$
\mathbf{f}_{2}(\mathbf{x}, \mathbf{y}, \boldsymbol{\delta})=\sum_{i=1}^{n} \Delta \mathbf{f}_{2 i}\left(\mathbf{x}, \mathbf{y}, \delta_{i}\right)
$$

where $n$ is the number of the control surfaces.

Figures from 7 to 9 present examples of the roll $L$, pitch $M$, and yaw $N$ moments produced by the right aileron $A R$, elevator $E R$ and flap $F R$ at a constant angle of attack equal to 1.5 degrees. It can be seen that the single elevator has the greatest influence on the pitch moment, and the single aileron and flap produces the greatest roll moments, as expected. What is more, the elevator influence on the roll and yaw moments is very low, and the aileron and the flap produce quite significant pitch and roll moments.

\section{Investigation of the control surfaces decoupling scheme}

The nonlinear UAV dynamic model with decoupled control surfaces has been tested. The tests were aimed at the investigation of the influence of operation of the decoupled control surfaces on the aircraft behavior during normal smooth flight. The results show a significant changes in aircraft behavior due to asymmetric control surfaces deflection. Deflecting any single control surface causes three dimensional changes of the aircraft attitude and the trajectory which requires immediate correction using other surfaces to achieve the demanded control results. That indicates that the lock of one of the control surfaces at any arbitrary position causes an unpredicted and unexpected response of the UAV aircraft to the control inputs, what may lead to 


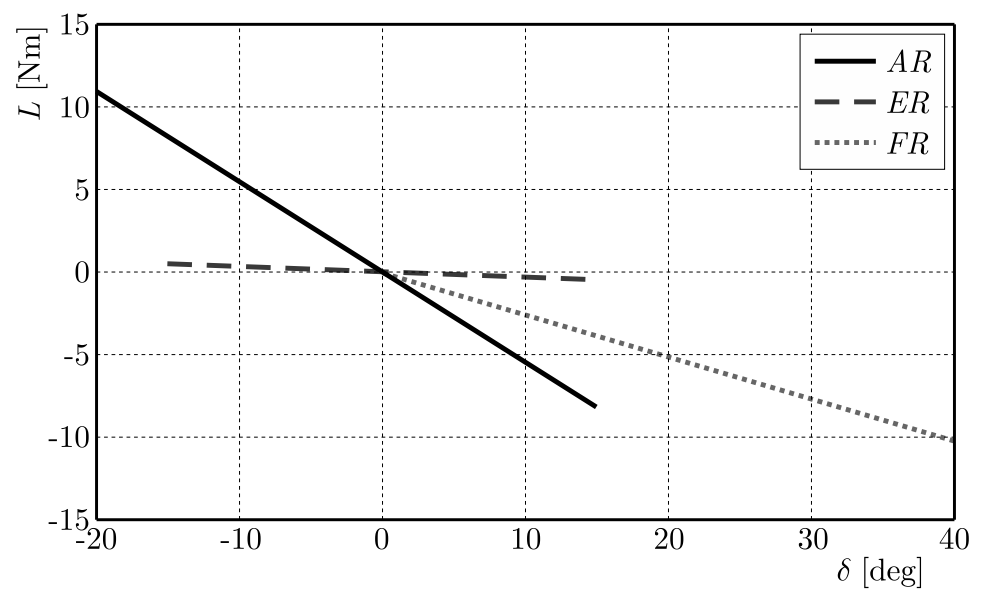

Fig. 7. Roll moment increment due to control surfaces deflection

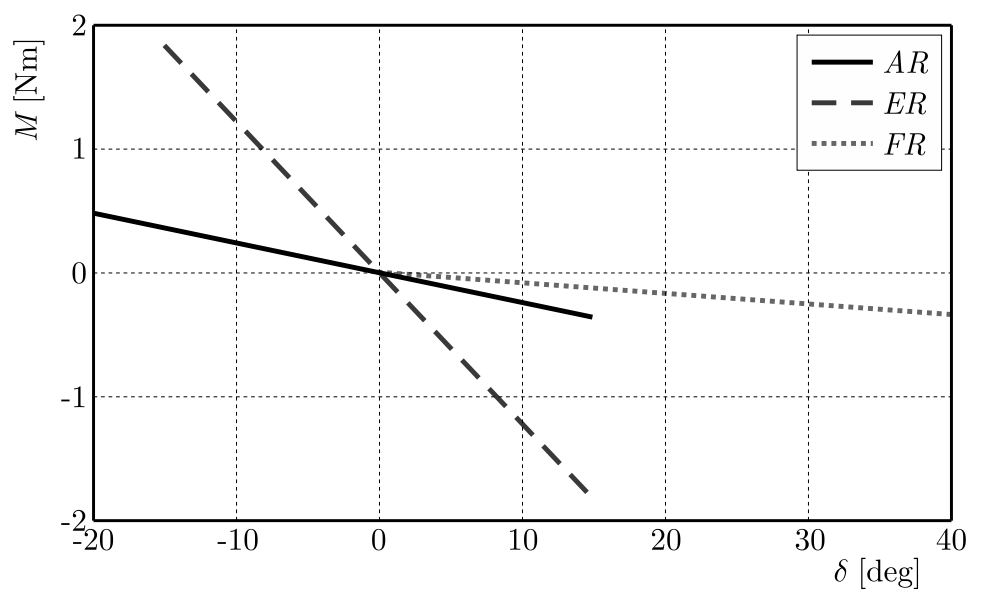

Fig. 8. Pitch moment increment due to control surfaces deflection

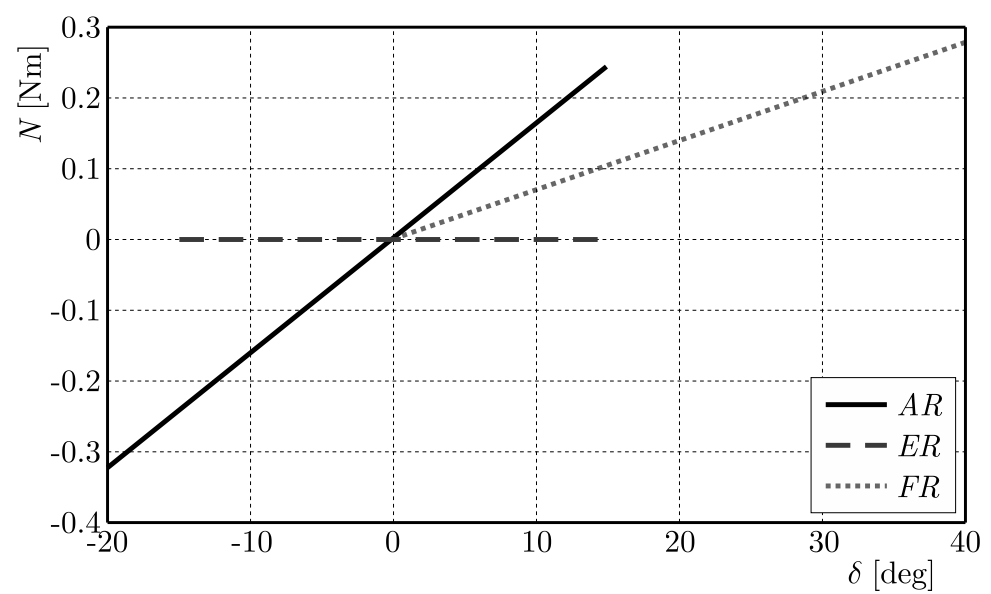

Fig. 9. Yaw moment increment due to control surfaces deflection

crash of the aircraft. What is more, if the control surface is locked in a non-neutral position (non zero deflection angle), these adverse effects will have significant influence on the equilibrium conditions of the aircraft.

Figures from 10 to 13 present a comparison of the aircraft behavior in two cases: the fault free configuration and the right elevator lock on the neutral position configuration. These tests 


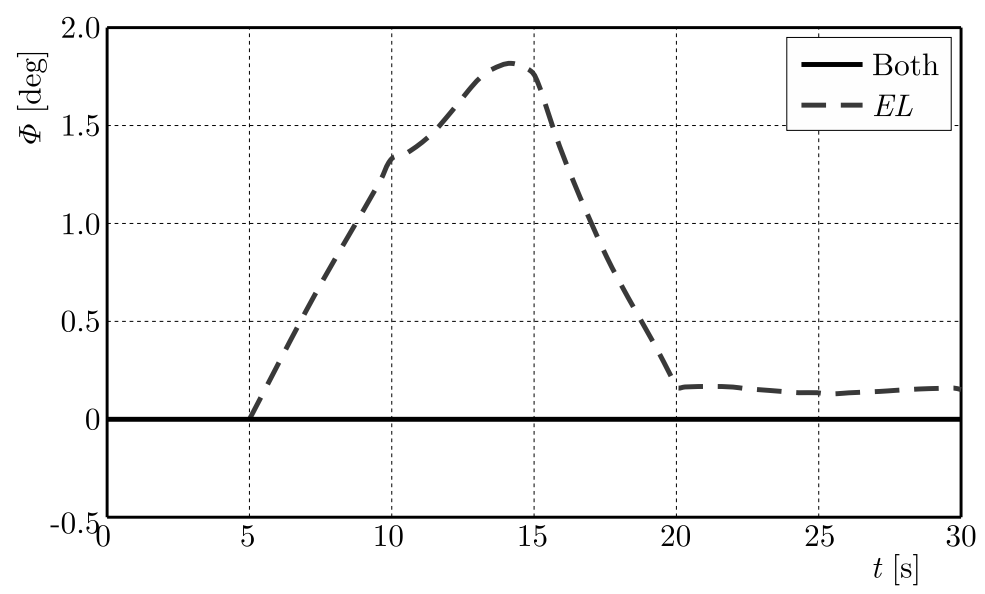

Fig. 10. Aircraft bank angle

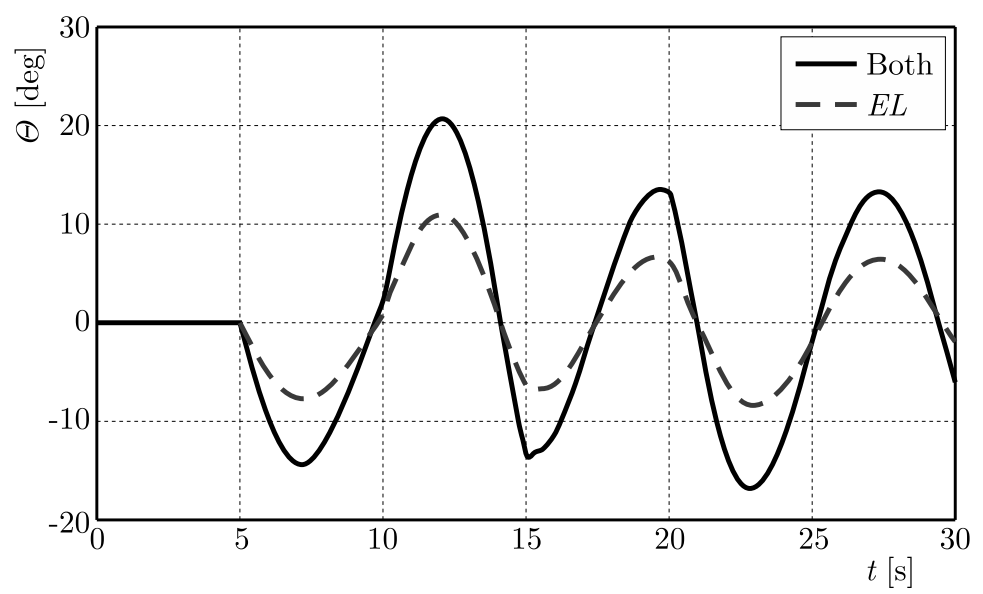

Fig. 11. Aircraft pitch angle

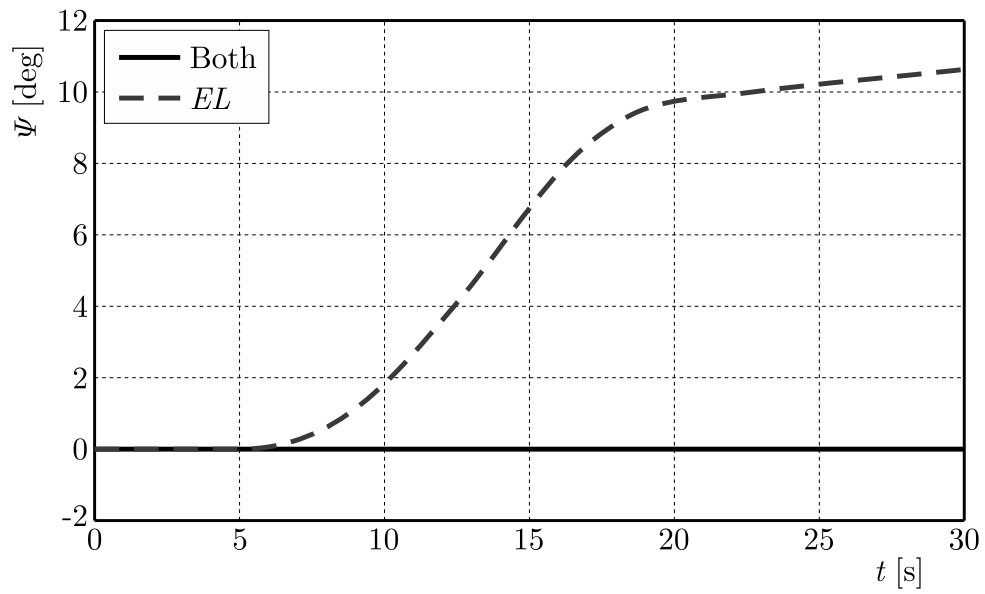

Fig. 12. Aircraft yaw angle

were performed for steady cruise conditions and the tasks were to apply a double impulse control signal to the elevators. At first, the control surfaces worked in the normal (coupled) configuration (both elevators operate), next the decoupled scheme was tested and the input signal was applied only to the left elevator. Only a phugoid oscillation was induced in the case of the normal configuration, what is typical. The phugoid oscillation had lower amplitude in the case of right elevator fault. What is more, the asymmetric elevator deflection induced disturbances to the bank and yaw angles. 


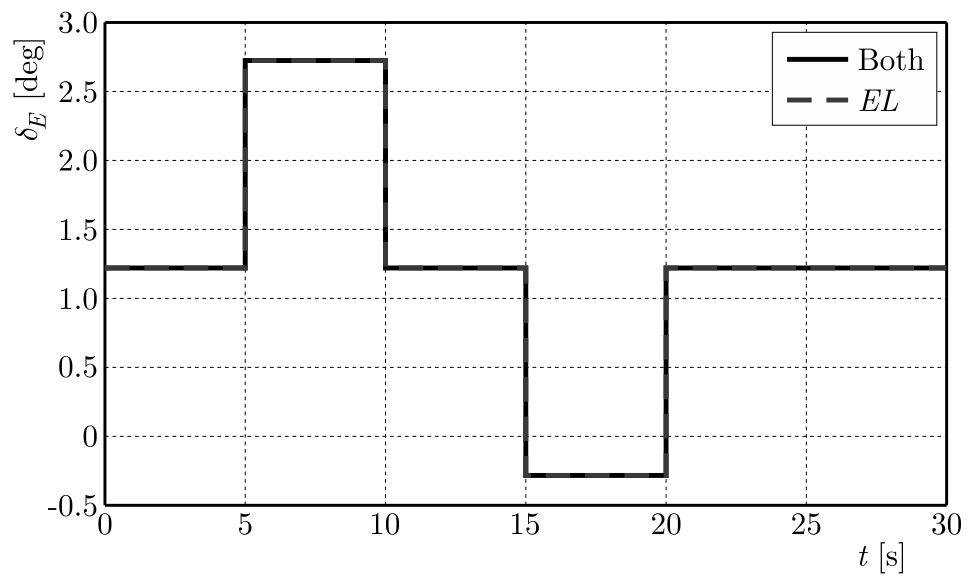

Fig. 13. Input signal

The UAV dynamic model has also been used to investigate the decoupled control system ability to reconfigure in the case of the lock of control surfaces. The main task of the control reconfiguration is at least to continue the steady flight after failure. The behavior of a damaged aircraft can be similar to the behavior of a failure-free aircraft, when the reconfigured control system is able to produce similar aerodynamic moments (Żugaj and Narkiewicz, 2010). The efficiency of the reconfiguration strongly depends on the control system redundancy.

The control system of the presented UAV aircraft consists of seven control surfaces: lefts aileron $A L$, elevator $E L$ and flap $F L$, and rights aileron $A R$, elevator $E R$ and flap $F R$, and the rudder $R$. The contribution of each control surface to the roll $L$, pitch $M$ and yaw $N$ aerodynamic moments is presented in Fig. 14. These figures present the proportion of the maximum positive and negative values of produced moments to the maximum values of moments in the normal configuration. It can be seen that the roll moment (of positive or negative value) can be generated by both ailerons and the flap. The contribution of each of these surfaces is almost equal. The pitch moment can be mainly generated by both elevators with a little contribution of both ailerons and flaps, but the flaps can produce only negative value of the pitching moment because of their handling limitations (flaps can be deflected down only). The control redundancy is very poor in the case of the yaw moment. It can be generated only by the rudder, and the contribution of other surfaces is not significant.
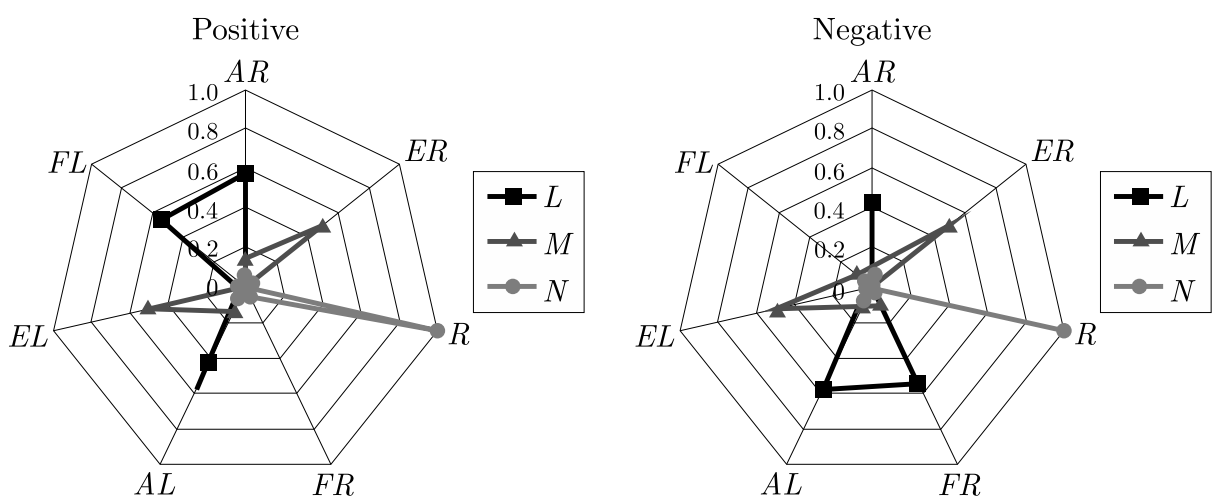

Fig. 14. Contributions of control surfaces to aerodynamic moments

Figure 15 presents the proportions (relative to the normal control configuration) of the total positive and negative values of the control moments produced by all control surfaces. It shows the advantages of the decoupled control system. The amount of the roll moment can be increased by about $60 \%$, the pitch moment by $30 \%$ and the yaw moment by $13 \%$ through handling all 
control surfaces individually. The amount of pitch moment increment could be increased by redesigning the flaps mechanization and extending their deflection range to an upper position (negative deflection angle), which could be done easily in the case of a small UAV airplane.

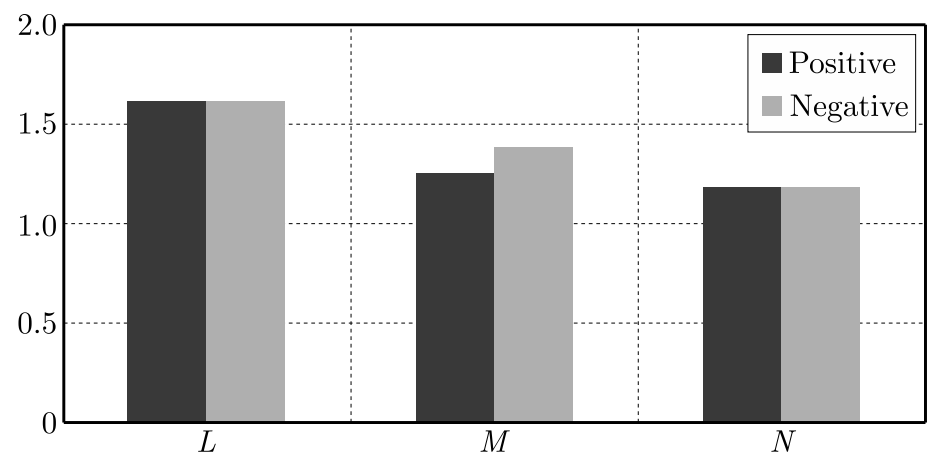

Fig. 15. Total values of aerodynamic moments produced by all control surfaces

\section{Conclusions}

The methodology of determination of the individual control surface aerodynamic loads is presented. A six degrees of freedom nonlinear model of the UAV aircraft with decoupled control surfaces has been developed. The influence of control surfaces failures on the UAV flight performances has been investigated using the model. The analysis of decoupled control system efficiency for reconfiguration has been performed as well.

The simulation results prove a significant influence of the control surface lock on the aircraft dynamic performances. The analysis of the decoupled control system indicates its ability to reconfigure, which could refine the aircraft reliability.

\section{Acknowledgments}

The research presented has been part of the project "Methods of synthesis of aircraft control system in emergency situations", under grant form National Centre for Research and Development, PBS2/B6/19/2013.

\section{References}

1. Bodson M., 2003, Reconfigurable nonlinear autopilot, Journal of Guidance Control and Dynamics, 26, 5, 719-727

2. Burcham B., 1997, Landing safely when flight controls fail, Aerospace America, 20-23

3. Cook M.V., 2007, Flight Dynamics Principles, Elsevier, Butterworth-Heinemann, Oxford

4. Defense Science Board Study on Unmanned Aerial Vehicles and Uninhabited Combat Aerial Vehicles, February 2004

5. Hass R.A., Wells S.R., 2003, Sliding mode control applied to reconfigurable flight control design, Journal of Guidance Control and Dynamics, 26, 3, 452-461

6. Kozak V.M., Shevchuk D.O., Vovk V.G., Levchenko M.H., 2014, Automation of aircraft control reconfiguration in flight special situations, Proceedings of IEEE 3rd International Conference on Methods and Systems of Navigation and Motion Control, 161-164

7. Lin X., Fulton N.L., Horn M.E.T., 2014, Quantification of high level safety criteria for civil unmanned aircraft systems, Proceedings of Aerospace Conference, Big Sky, 1-13 
8. Loh R., Bian Y., Roe T., 2006, Safety requirements for unmanned aerial vehicles (UAV) in future civil airspace, Proceedings of Position, Location, and Navigation Symposium, 1151-1163

9. Loh R., Bian Y., Roe T., 2009, UAVs in civil airspace: safety requirements, IEEE Aerospace and Electronic Systems Magazine, 24, 1, 5-17

10. Masui K., Tomita H., Komatsu Y., 2004, Flight experiment on flight path optimization algorithm for aircraft in trouble, Proceedings of 24 th International Conference of the Aeronautical Science

11. NizioŁ J., 2005, Dynamics of Mechanical Systems (in Polish), Komitet Mechaniki PAN, Instytut Podstawowych Problemów Techniki Polskiej Akademii Nauk, Warszawa

12. Suzuki S., Kawamura F., Masui K., 2004, Autonomous flight control and guidance system of accident aircraft, Proceedings of 24 th International Conference of the Aeronautical Science

13. Uhlig D., Bhamidipati K., Neogi N., 2006, Safety and reliability within UAV construction, Proceedings of 25th Digital Avionics Systems Conference

14. Young A.D., 1953, The Aerodynamics Characteristics Of Flaps, A.R.C. Technical Report No. 2622 (10766), Her Majesty's Stationery Office

15. ŻugaJ M., NARKIEWICZ J., 2007, Impact of control system degradation on aircraft flight performance (in Polish), Zeszyty Naukowe Politechniki Rzeszowskiej, Mechanika, 71, 179-186

16. Żugaj M., NARKIEWICZ J., 2009, Autopilot for reconfigurable flight control system, ASCE Journal of Aerospace Engineering, 22, 1, 78-84

17. ŻUgAJ M., NARKIEwICZ J., 2010, Autopilot supported by nonlinear model following reconfigurable flight control system, ASCE Journal of Aerospace Engineering, 22, 1, 339-347

Manuscript received November 3, 2015; accepted for print April 14, 2016 